\title{
Modelagem da qualidade das águas a partir de sensoriamento remoto hiperespectral ${ }^{1}$
}

\author{
Fernando B. Lopes², Cláudio C. F. Barbosa ${ }^{3}$, Evlyn M. L. de M. Novo ${ }^{4}$, \\ Eunice M. de Andrade ${ }^{5}$ \& Luiz C. G. Chaves ${ }^{6}$

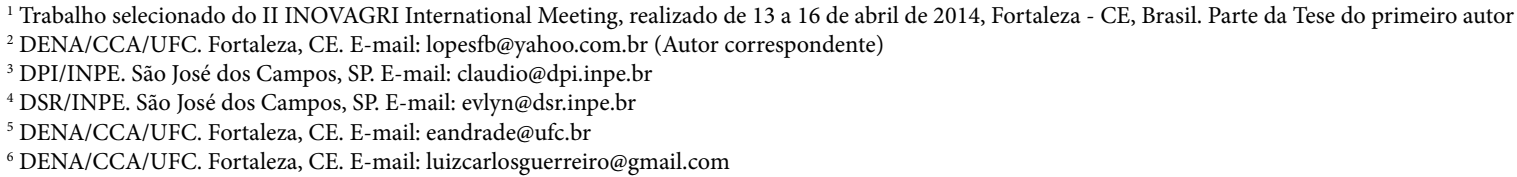

\section{Palavras-chave:}

região semiárida

monitoramento da qualidade da água

sensor hiperespectral

\begin{abstract}
R E S U M O
Este estudo objetiva estimar as variáveis limnológicas a partir de dados de sensoriamento remoto. Os dados foram coletados em 20 pontos no reservatório Orós, Ceará, em março de 2011 e agosto de 2012. Foram analisados os atributos: transparência de Secchi, turbidez, sedimentos inorgânicos em suspensão (SIS), condutividade elétrica da água (CE) e dados radiométricos. Posteriormente, foram realizadas análises de correlação entre o fator de reflectância bidirecional - FR e os dados de SIS, CE, turbidez e transparência e gerados os modelos de regressão simples. Para o atributo SIS o modelo ajustado foi o potencial ( $\mathrm{SIS}=860,1679{ }^{*} \mathrm{FR}_{\lambda 720}{ }^{1 / 0,6427}$ ), com um coeficiente de determinação $\left(R^{2}\right)$ de 0,90 . Para a variável turbidez, o modelo de regressão ajustado foi Turb $=\left(\left(\mathrm{FR}_{\lambda 720}-0,0217\right) / 0,0017\right)$ e com $\mathrm{R}^{2}$ de 0,90 . O modelo de regressão ajustado para a transparência foi Transp $=\left(\left(-\mathrm{FR}_{\lambda 653}+0,171\right) / 0,1375\right)$ e com $\mathrm{R}^{2}$ de 0,92 . O modelo de regressão ajustado para a condutividade elétrica foi $C E=\left(\left(-\mathrm{FR}_{\lambda 632}+0,5352\right) / 1,6278\right)$ e com $\mathrm{R}^{2}$ igual a 0,93 . Para todos os modelos os valores de $\mathrm{p}$ (p-value) foram menores que 0,001 . Os modelos desenvolvidos indicam que as variáveis limnológicas podem ser quantificadas remotamente.
\end{abstract}

Key words: semi-arid region monitoring of water quality hyperspectral sensor

\section{Water quality model using hyperspectral remote sensing}

\begin{abstract}
A B S T R A C T
This study aimed to estimate limnological parameters using remote sensing data. Data were sampled in 20 sites in the Orós reservoir in Ceará State, Brazil in March/2011 and August/2012. Secchi transparency, turbidity, suspended inorganic sediments (SIS), transparency, electrical conductivity of water (CE), and radiometric data were analysed. After sampling the data were submitted to correlation and regression analysis between the bidirectional reflectance factor (FR) and SIS, CE, turbidity and transparency data. For estimating SIS the adjusted model was the potential model (SIS $=860.1679^{\star} \mathrm{FR}_{\lambda 720}{ }^{1 / 0.6427}$ ) with a coefficient of determination $\left(\mathrm{R}^{2}\right)$ of 0.90 . For turbidity the best model was represented by euqation Turb $=\left(\mathrm{FR}_{\lambda 720}-0.0217\right) / 0.0017$ providing a $\mathrm{R}^{2}$ of 0.90 and the best model for transparency was Transp $=\left(-\mathrm{FR}_{\lambda 653}+0.171\right) / 0.1375$ with an $\mathrm{R}^{2}$ of 0.92 . For the electrical conductivity of water the $\mathrm{CE}=\left(-\mathrm{FR}_{\lambda 632}\right.$ $+0.5352) / 1.6278$ was the best with a $\mathrm{R}^{2}$ of 0.93 . For all these models, $\mathrm{p}$-value was smaller than 0.001 . These models seems to be reliable and indicate that these limnological variables can be estimated using in situ remote sensing data.
\end{abstract}

\section{INTRODUÇÃo}

A água é essencial para todas as formas de vida. De acordo com a UNESCO (2012) calcula-se que a população urbana mundial deve aumentar de 3,4 bilhões para 6,3 bilhões de pessoas, no período entre 2009 e 2050. Na medida em que cresce a demanda de recursos hídricos no mundo, diminui a probabilidade do fornecimento de água doce. Como agravante, nas últimas décadas a qualidade da água vem sendo rapidamente deteriorada o que inviabiliza a utilização de importantes reservatórios naturais como os de água subterrânea e mananciais ou os antropogênicos, como os reservatórios hidroelétricos e açudes (Alexandre et al., 2010; Li et al., 2011; Guedes et al., 2012).
Com o crescimento da população, aliado às altas taxas de consumo de água, ao modelo de desenvolvimento adotado e à contaminação dos recursos hídricos pela ação antrópica, a tendência é de que os recursos hídricos se tornem mais escassos, qualitativa e quantitativamente, caso não haja ações enérgicas visando à melhoria da gestão da oferta e da demanda da água para os diferentes usos. Portanto, manter o abastecimento d’água, não apenas em quantidade, mas também em qualidade, é um grande desafio a ser superado pela sociedade.

O manejo adequado dos recursos hídricos requer um conjunto de informações em um contexto dinâmico sobre o funcionamento destes sistemas no interior da bacia hidrográfica. Assim, é imprescindível um manejo integrado dos reservatórios 
para o monitoramento da qualidade da água, pois, o mesmo permite identificar, além das variações espaço-temporais, a relação entre os diversos mecanismos dos sistemas hídricos e a totalidade de sua bacia de drenagem (Jong et al., 1995; Silva et al., 2009).

O monitoramento da qualidade da água nos dias atuais é essencial, e os dados de sensoriamento remoto podem tornalo mais bem sucedido. O uso de dados de sensoriamento remoto apresenta grande potencialidade para a identificação da qualidade da água, permitindo o monitoramento em diferentes escalas espacial e temporal.

Com o sensoriamento remoto é possível avaliar as respostas decorrentes de perturbações introduzidas pela atividade humana, de modo a prever o impacto dessas ações sobre suas condições de sustentabilidade em médio e longo prazo (Novo, 2005). Essas técnicas podem ser usadas de modo eficiente para prevenir, constatar e monitorar mudanças ocorridas no sistema aquático (Dekker et al., 1992; Novo, 2005).

Os efeitos espectrais dos componentes opticamente ativos (COAs) sobre a reflectância da água têm sido amplamente discutidos na literatura (Chen et al., 1992; Han \& Rundquist, 1997; Lodhi et al., 1997; Rudorff et al., 2007; Gitelson et al., 2011; Pereira et al., 2011; Le et al., 2013).

Neste sentido, o estudo dos COAs presentes na água usando dados radiométricos é relevante, uma vez que permite a avaliação de problemas dinâmicos, tais como a eutrofização de corpos de água e dos sedimentos em suspensão (Lopes et al., 2013). Portanto, objetivou-se estimar as concentrações de sedimentos inorgânicos em suspensão - SIS, turbidez, transparência e da condutividade elétrica da água - CE utilizando-se os dados de sensoriamento remoto hiperespectrais de campo.

\section{MAterial e Métodos}

A área de estudo corresponde ao açude Orós, o principal reservatório da bacia do Alto Jaguaribe, região Centro Sul do Estado do Ceará (Figura 1). A barragem do reservatório foi projetada e concluída, em 1961, pelo Departamento Nacional de Obras Contra as Secas - DNOCS, com a finalidade de perenizar o rio Jaguaribe visando o abastecimento da população (regiões do Jaguaribe e Metropolitana de Fortaleza, capital do Estado do Ceará), o desenvolvimento da agricultura irrigada, da piscicultura e do turismo (DNOCS, 2014). O açude Orós (Figura 1) tem capacidade de armazenamento na cota de sangria de 1,9 bilhões de $\mathrm{m}^{3}$ e espelho de água de aproximadamente $190 \mathrm{~km}^{2}$.

Conforme a classificação de Köppen, a região apresenta clima do tipo BSw'h', semiárido quente com precipitações máximas de outono, e temperatura média mensal sempre superior a $18^{\circ} \mathrm{C}$; com precipitação pluviométrica média anual (1974 a 2011) de $998 \pm 321 \mathrm{~mm}$, sendo a precipitação pluviométrica na região concentrada principalmente nos meses de janeiro a maio; a evaporação medida através de Tanque Classe A, está em torno dos $1.988 \mathrm{~mm}$ ano $^{-1}$; a umidade relativa do ar $66,1 \%$, com ventos a uma velocidade de $1,8 \mathrm{~m} \mathrm{~s}^{-1}$ e insolação de $2.945 \mathrm{~h} \mathrm{ano}^{-1}$.

Foram determinados os seguintes atributos do reservatório: concentrações de sedimentos inorgânicos em suspensão - SIS

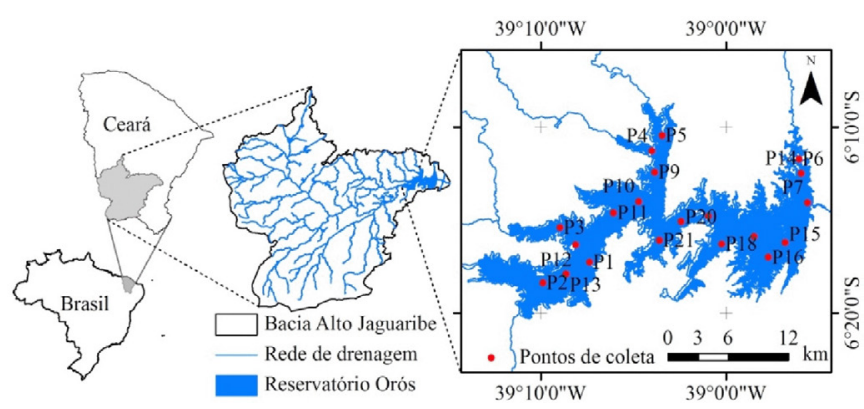

Figura 1. Localização do reservatório Orós, Ceará, com os pontos de coleta

(mg L $\left.{ }^{-1}\right)$, turbidez (NTU), transparência (m) e condutividade elétrica da água - CE $\left(\mathrm{dS} \mathrm{m}^{-1}\right)$. Simultaneamente foram também realizadas medidas da radiância da água nos meses de março de 2011 e agosto de 2012, em 20 estações amostrais (Figura 1).

As medições da radiância espectral do sistema aquático foram realizadas utilizando o espectrorradiômetro ASD FieldSpec 3 Hi-Res (Figura 2), com campo de visada de $25^{\circ}$, resolução espectral de 1,4 nm, e que abrange a faixa espectral de 350 a $2500 \mathrm{~nm}$. Também foi utilizada uma placa de referência de Spectralon, representando uma superfície lambertiana. As medições foram realizadas entre 10 e 14 horas, no período de menor ângulo de inclinação solar, maior fluxo de energia radainte e condições meteorológicas adequadas, com pouco vento e céu predominantemente livre de nuvens.

As medidas radiométricas foram coletadas seguindo os procedimentos da metodologia proposta por Milton (1987), ou seja, preservou ao máximo a igualdade de condições de iluminação e de superfície de água. O espectrorradiômetro foi posicionado em cada ponto amostral com eixo de visada de $40^{\circ}$ de inclinação em relação à vertical e de $90^{\circ}$ de azimute em relação à posição do Sol, minimizando a reflectância especular da água. A altura do sensor em relação à superfície da água foi de $1 \mathrm{~m}$ e o tamanho aproximado da área imageada foi de 0,7 $\mathrm{m}^{2}$ (Figura 2).

Para cada ponto foram realizadas 10 medidas de radiância da água, para que posteriormente fossem eliminadas as medidas não representativas e calculada a média para as restantes. Os valores de radiância espectral foram convertidos em fator de reflectância bidirecional conforme a Eq. 1:

$$
\operatorname{FRB}_{\lambda} \quad \mathrm{L}_{\mathrm{a}, \lambda} / \mathrm{L}_{\mathrm{r}, \lambda}
$$

em que:

$\mathrm{FRB}_{\lambda}$ - fator de reflectância bidirecional (adimensional)

$\mathrm{L}_{\mathrm{a}, \lambda}$ - radiância espectral do alvo $\left(\mathrm{W} \mathrm{cm}^{-2} \mathrm{sr}^{-1} \mu \mathrm{m}^{-1}\right)$

$\mathrm{L}_{\mathrm{r}, \lambda}$ - radiância espectral da placa de referência $\left(\mathrm{W} \mathrm{cm}^{-2}\right.$ $\left.\mathrm{sr}^{-1} \mu \mathrm{m}^{-1}\right)$

O cálculo da regressão linear foi efetuado por meio de processamento eletrônico dos dados, empregando-se o software estatístico SPSS (Statistical Package for Social Sciences) versão 16.0 (Norusis, 1990). Utilizando-se o SPSS, foram obtidos os valores dos coeficientes (angular e linear) da equação e o coeficiente de determinação $\left(\mathrm{R}^{2}\right)$. 

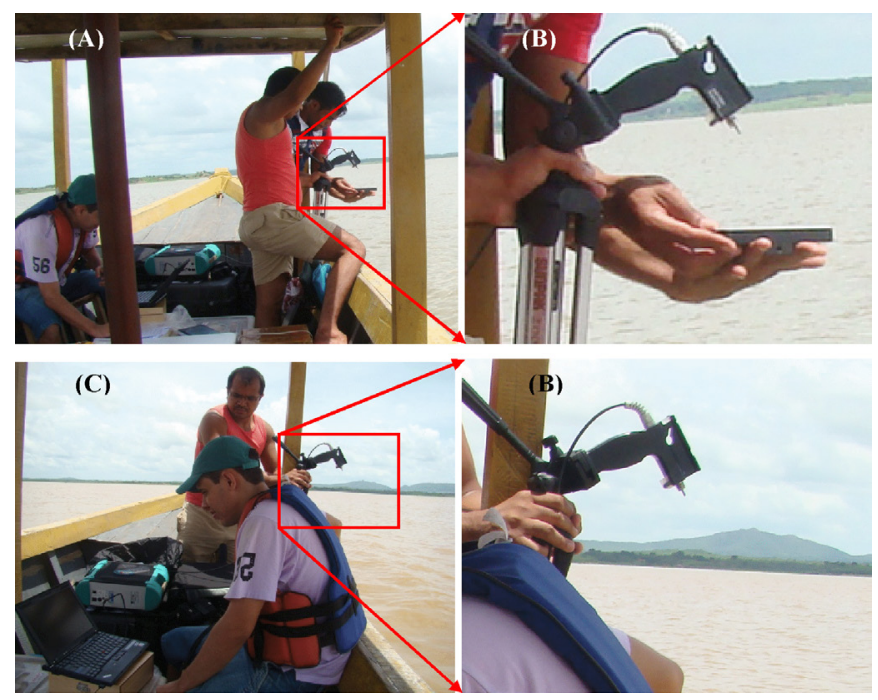

Figura 2. Leitura da placa lambertiana (A), zoom da leitura da placa (B), leitura da radiância espectral do sistema aquático (C), zoom da leitura da radiância espectral do sistema aquático (D) procedimentos realizados para a coleta de dados radiométricos no reservatório Orós, Ceará

Para o cálculo dos modelos de regressão, foram utilizados os dados de março de 2011, em seguida foi feita a validação dos modelos empregando-se dados referentes de agosto de 2012, evitando-se com isso falhas proporcionadas pela autocorrelação dos dados, o que poderia induzir o modelo a resultados tendenciosos.

Para avaliar estatisticamente o desempenho dos modelos, calcularam-se indicadores estatísticos comparando-se os valores estimados e os medidos não empregados na análise de regressão. Os indicadores estatísticos utilizados nessa avaliação foram: coeficiente de correlação $(\mathrm{r})$; coeficiente de determinação $\left(\mathrm{R}^{2}\right)$; teste F; índice de Willmott (d); coeficiente de Nash-Sutcliffe (NSE); erro médio absoluto (EMA) e raiz do erro médio quadrático (REMQ).

O coeficiente de correlação de Pearson é uma medida do grau de relação linear entre duas variáveis quantitativas. Este coeficiente varia entre os valores - 1 e 1 . O valor 0 (zero) significa que não há relação linear, o valor 1 indica uma relação linear perfeita e o valor - 1 também indica uma relação linear perfeita, mas inversa, ou seja, quando uma das variáveis aumenta a outra diminui. Quanto mais próximo estiver de 1 ou -1, mais forte é a associação linear entre as duas variáveis (Tabela 1).

A precisão é dada pelo coeficiente de correlação que indica o grau de dispersão dos dados obtidos em relação à média, ou seja, o erro aleatório. O coeficiente de determinação, $\mathrm{R}^{2}$, é a

Tabela 1. Interpretação dos coeficientes de correlação e determinação de Pearson

\begin{tabular}{ccc}
\hline & Escala de Pearson & \\
\hline Fraca & Moderada & Forte \\
$0 \leq r \leq 0,50$ & $0,50 \leq r \leq 0,90$ & $0,90 \leq r \leq 1,00$ \\
$0 \leq r^{2} \leq 0,25$ & $0,25 \leq r^{2} \leq 0,81$ & $0,81 \leq r^{2} \leq 1,00$ \\
\hline
\end{tabular}

Fonte: Adaptado de Milton (1992) porcentagem da variação da variável dependente explicada pela variável(eis) independente(s), Tabela 1.

O índice de Willmott (d) foi calculado pela Eq. 2 (Willmott et al., 1985), cujos valores variam de zero, para nenhuma concordância, a 1, para a concordância perfeita. Valores de d acima de 0,75 são considerados satisfatórios.

$$
\mathrm{d}=1-\frac{\sum(\mathrm{Pi}-\mathrm{OI})^{2}}{\sum(|\mathrm{Pi}-\mathrm{O}|+|\mathrm{Oi}-\mathrm{O}|)^{2}}
$$

em que:

$$
\begin{array}{ll}
\mathrm{d} & \text { - é o índice de concordância de Willmott } \\
\mathrm{Pi} & \text { - expressa o valor estimado da variável } \\
\mathrm{Oi} & \text { - representa o valor observado } \\
\mathrm{O} & \text { - define a média dos valores observados }
\end{array}
$$

O coeficiente definido por Nash \& Sutcliffe (1970) traduz a similaridade da variabilidade entre duas varáveis; é um indicador da similaridade de quantificação, considerado relevante na avaliação de confiança e eficiência de modelos e é um critério estatístico importante para avaliar a precisão de modelos (Machado et al., 2003). Foi determinado pela Eq. 3:

$$
\mathrm{NSE}=1-\left[\frac{\sum\left(\mathrm{Y}_{\mathrm{m}}-\mathrm{Y}_{\mathrm{c}}\right)^{2}}{\sum\left(\mathrm{Y}_{\mathrm{m}}-\overline{\mathrm{Y}}_{\mathrm{m}}\right)^{2}}\right]
$$

em que:

$$
\begin{aligned}
& \text { NSE - coeficiente de Nash e Sutcliffe, }(-\infty<\mathrm{NS} \leq 1) \\
& \text { Ym - valor medido } \\
& \text { Yc - valor calculado } \\
& \mathrm{Y}_{\mathrm{m}} \quad \text { - média dos valores medidos }
\end{aligned}
$$

Este coeficiente pode variar de - $\infty$ até 1 , sendo 1 um ajuste perfeito. O desempenho de um modelo é considerado adequado e bom se o valor de NSE superar 0,75, e é considerado aceitável se o valor de NSE fica entre 0,36 e 0,75 . Quando o coeficiente NSE apresentar valores menores que zero indica que o valor médio da série de dados em estudo é um melhor indicador que a estimativa usando o modelo.

O erro médio absoluto (EMA) é definido como sendo a diferença entre a previsão e a observação, dividida pelo número de observações (Eq. 4):

$$
\mathrm{EMA}=\frac{1}{\mathrm{~N}} \sum_{\mathrm{i}=1}^{\mathrm{N}}\left|\mathrm{X}_{\mathrm{i}}^{\prime}-\mathrm{X}_{\mathrm{i}}\right|
$$

em que:

$\mathrm{X}_{\mathrm{i}}$ - dados medidos

$\mathrm{X}_{\mathrm{i}}$ - dados estimados

$\mathrm{N}$ - número pares de pontos utilizados. Quando uma previsão for perfeita o EMA será igual a zero. 
A raiz do erro médio quadrático (REMQ) foi obtida pela Eq. 5. Este método indica o grau de similaridade entre os dados medidos e os estimados usando os modelos, sendo o valor ideal igual a zero. O REMQ é mais sensível a valores extremos.

$$
\operatorname{REMQ}=\sqrt{\frac{1}{N} \sum_{i=1}^{N}\left[X_{i}^{\prime}-X_{i}\right]^{2}}
$$

\section{Resultados e Discussão}

Para identificar as posições espectrais de maior correlação com as variáveis limnológicas, a reflectância espectral das estações amostradas em março de 2011 no reservatório Orós foram construídos correlogramas expressando a correlação entre o FRB e as variáveis: SIS ( $\left.\mathrm{mg} \mathrm{L}^{-1}\right)$, turbidez (UNT), transparência $(\mathrm{m})$ e $\mathrm{CE}\left(\mathrm{dS} \mathrm{m}^{-1}\right)$, Figuras 3 .

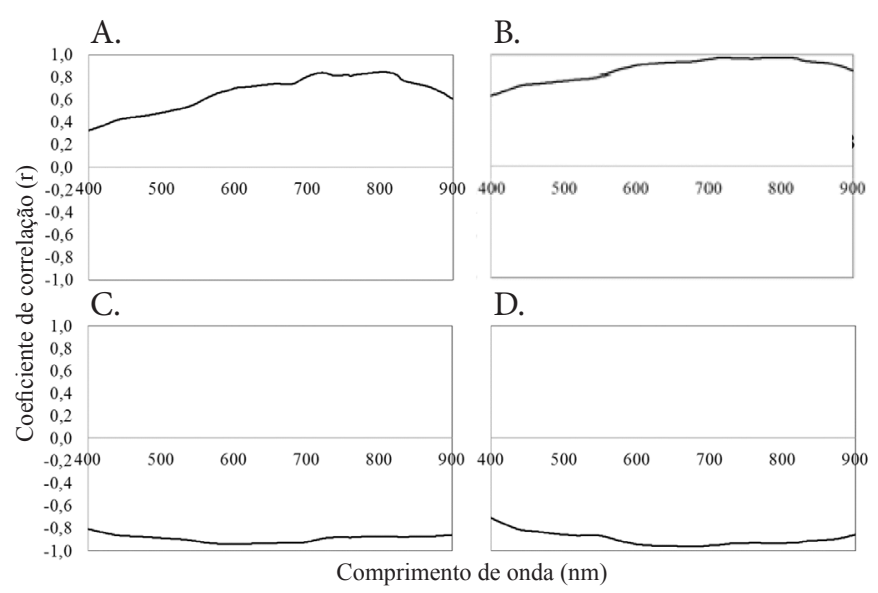

Figura 3. Correlogramas entre o fator de reflectância e variáveis medidas no reservatório Orós, Ceará: (A) SIS em mg L-1, (B) turbidez em UNT, (C) transparência em $\mathrm{cm}$ e (D) condutividade elétrica - CE, em dS $\mathrm{m}^{-1}$

As bandas espectrais, cujos valores do fator de reflectância apresentaram os melhores resultados de correlação (Figura 3), foram testadas para estimar as variáveis: Sólidos inorgânicos suspensos - SIS, turbidez, transparência e condutividade elétrica - CE (Figura 4).

O comprimento de onda de $720 \mathrm{~nm}$ apresentou correlação positiva com a variável sólidos inorgânicos suspensos - SIS, sendo que o modelo potencial $\left(\mathrm{SIS}=860,1679^{*} \mathrm{FR}_{\lambda 720}{ }^{1 / 0,6427}\right.$ ) (Figura 4A) teve melhor desempenho que o modelo linear. Resultados semelhantes foram encontrados por Lodhi et al. (1997) que, pesquisando o potencial do sensoriamento remoto para estimativas das concentraçõe de sedimentos em suspensão em água superficiais, determinaram o modelo potencial com $\mathrm{R}^{2}$ de 0,96 em $855 \mathrm{~nm}$. Diferentemente das regressões lineares obtidas por Chen et al. (1992); Han \& Rundquist (1997) e Lopes et al. (2013) o ajuste da regressão entre os dados de sensoriamento remoto hiperespectrais de campo e as concentrações de sedimentos inorgânicos em suspensão foi não linear. Portanto, águas continentais da região semiárida (resevartório Orós), apresentam uma maior complexidade óptica. Resultados semelhantes foram encontrados por Rudorff
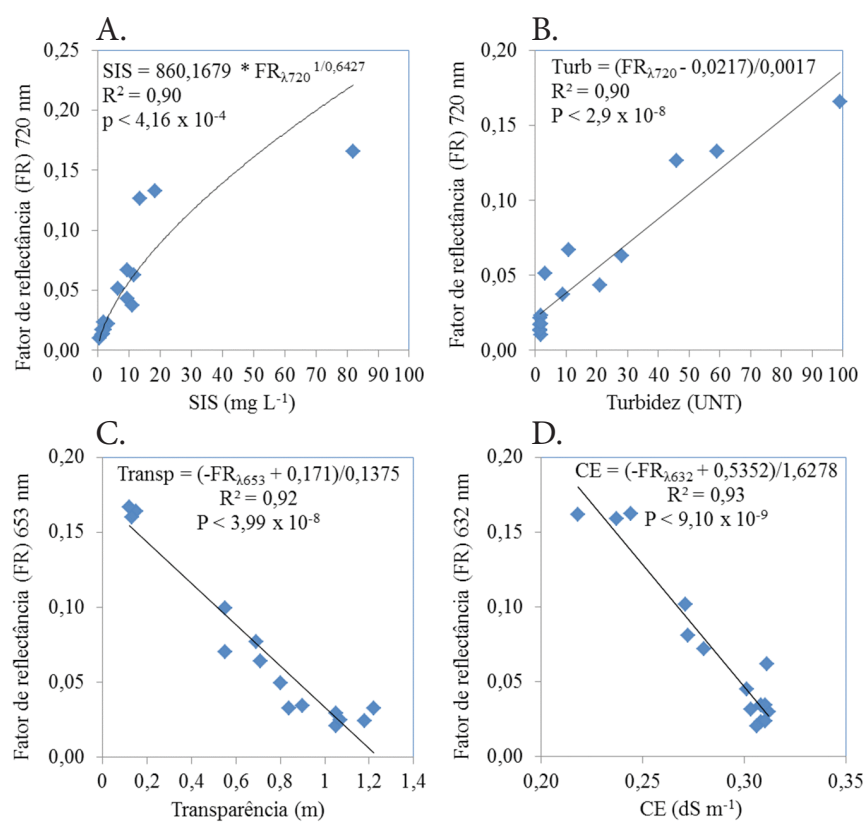

Figura 4. Modelos para estimativas das variáveis limnológicas (A) sólidos inorgânicos suspensos - SIS (B) turbidez, (C) transparência e (D) condutividade elétrica - CE

et al. (2007) caracterizando a composição de águas opticamente complexas na Amazônia.

Para a variável turbidez, o modelo de regressão ajustado Turb $=\left(\mathrm{FR}_{1720}-0,0217\right) / 0,0017$ apresentou coeficiente de determinação $\left(R^{2}\right)$ de 0,90 e p $<2,9 \times 10^{-8}$. À medida que aumenta a turbidez, o fator de reflectância em $720 \mathrm{~nm}$ também aumenta (Figura 4B). Resultados semelhantes foram encontrados por Barros et al. (2003) estimando a turbidez da água a partir de dados sensoriamento remoto.

O modelo de regressão ajustado para a transparência das águas do reservatório Orós foi, Transp $=\left(-\mathrm{FR}_{\lambda 653}+\right.$ $0,171) / 0,1375$ com um coeficiente de determinação $\left(\mathrm{R}^{2}\right)$ de 0,92 (Figura 4C), caracterizado como bom, de acordo com Milton (1992). Pereira et al. (2011) estudando a transparência da água no reservatório de Itupararanga, São Paulo, a partir de imagens multiespectrais IKONOS e espectrorradiometria de campo encontraram $\mathrm{R}^{2}$ ajustado explicando $47,8 \%$ da variabilidade dos dados.

O modelo de regressão ajustado para a condutividade elétrica das águas do reservatório Orós foi $\mathrm{CE}=\left(\left(-\mathrm{FR}_{\lambda, 632}+\right.\right.$ 0,5352)/1,6278), que apresentou coeficiente de determinação $\left(\mathrm{R}^{2}\right)$ igual a 0,93 (Figura $4 \mathrm{D}$ ). Resultados semelhantes foram encontrados por Choubey (1994) e Lopes et al. (2013), estimando a condutividade elétrica da água a partir de dados de sensoriamento remoto. Apesar da condutividade elétrica da água não ser um componente opticamente ativo e não ter uma assinatura espectral, mas no caso de Orós, ela co-varia com sedimentos em suspensão que é um componente opticamente ativo. A condutividade elétrica da água apresentou correlação com a turbidez de 0,95 e 0,77 para os sólidos inorgânicos suspensos. Lopes et al. (2013), observaram alta correlação entre as concentrações de sedimentos inorgânicos em suspensão e 
condutividade elétrica da água com coeficientes de $\mathrm{R}^{2}=0,95$ e $\mathrm{r}$ $=0,97$. Todas as correlações para os modelos foram significativas (Figura 4).

Para avaliar se os erros de estimativa apresentavam tendência, os resíduos dos modelos foram observados em gráficos de dispersão entre os dados de coleta in situ das concentrações das variáveis limnológicas SIS, turbidez, transparência e CE (Figura 5).
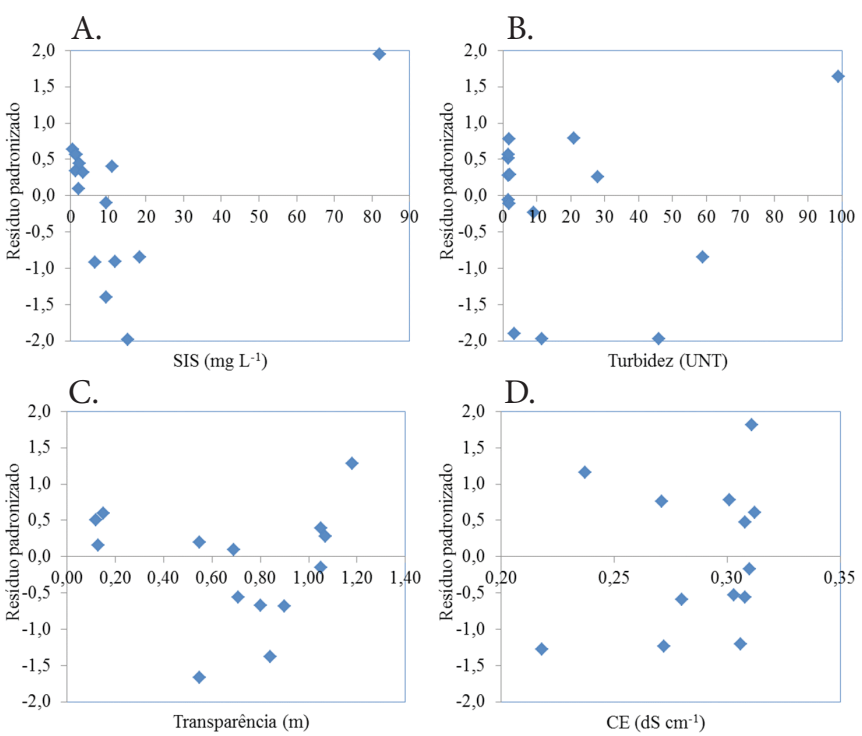

Figura 5. Dispersão entre os resíduos e as concentrações das variáveis limnológicas (A) sólidos inorgânicos suspensos - SIS (B) turbidez, (C) transparência e (D) condutividade elétrica - CE

Não foi observado padrão evidente de que haja aumento sistemático dos resíduos com o aumento ou diminuição nas concentrações destas variáveis estimadas, ou seja, os erros são distribuídos aleatoriamente (Figura 5). Verifica-se ainda que os resíduos distribuem-se aleatoriamente em torno de zero e que todos os erros dos resíduos estão entre -2 e 2 .

De acordo com o resultado obtido para as bandas selecionadas, os modelos (Figura 4) foram validados (Figura 6) com base em amostras coletadas in situ para a campanha de campo realizada em agosto de 2012 no reservatório Orós, Ceará.

Os parâmetros usados para analisar o desempenho do modelo para a variável SIS apresentam $\mathrm{R}^{2}$ de $0,83, \mathrm{r}$ de 0,91 , d de 0,79 e NSE 0,44, classificados, como forte para o $R^{2}$ e $r$, satisfatório para o d e aceitável para o NSE (Figura 6A). O modelo apresenta baixo erro de estimativa com EMA de 1,84 e RQEM de 2,08 ( $\mathrm{mg} \mathrm{L}^{-1}$ ). Rudorff et al. (2007) caracterizando a composição de águas opticamente complexas na Amazônia encontraram um modelo que resultou em um erro quadrático médio de $3,49 \mathrm{mg} \mathrm{L}^{-1}$.

O desempenho do modelo para a variável turbidez apresentou $\mathrm{R}^{2}$ de $0,91, \mathrm{r}$ de $0,95, \mathrm{~d}$ de 0,89 e NSE 0,81, classificados respectivamente, como forte para o $\mathrm{R}^{2}$ e $\mathrm{r}$, satisfatório e adequado (Figura 6B). O modelo apresenta erro de estimativa com EMA de 4,28 e RQEM de 5,03 (UNT). O
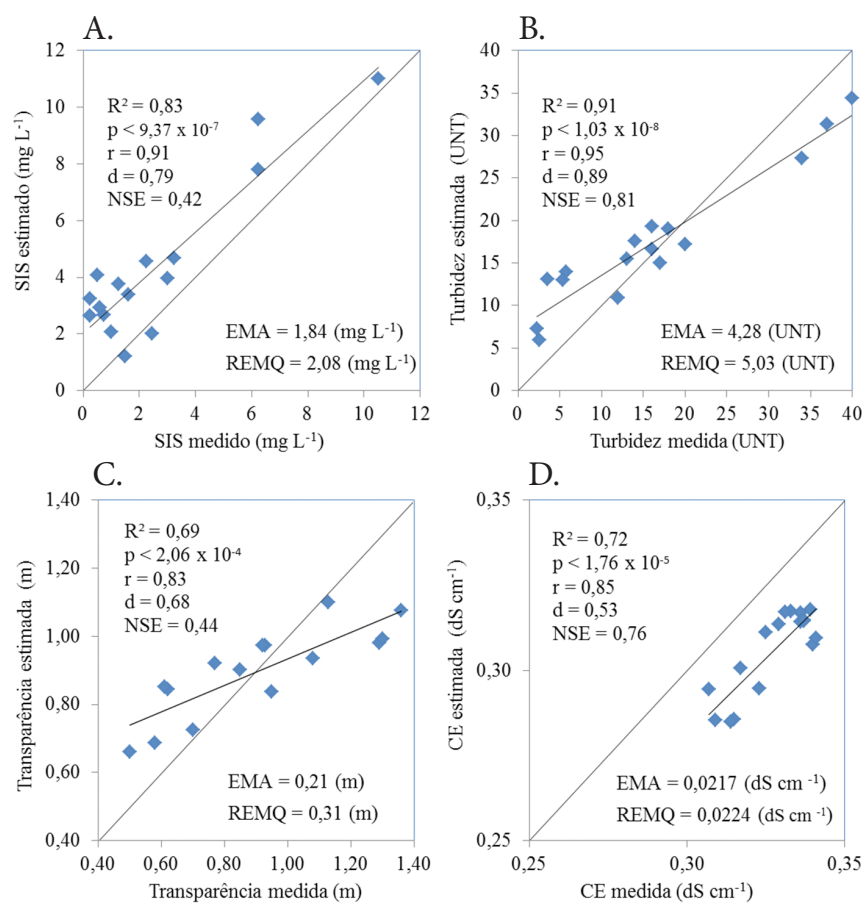

Figura 6. Validação dos modelos para quantificação das concentrações das variáveis limnológicas (A), sólidos inorgânicos suspensos - SIS (B), turbidez, (C) transparência e (D) condutividade elétrica - CE

modelo tende a superestimar os baixos valores e subestimar os valores altos de turbidez. Essa variável é influenciada pela presença de sólidos em suspensão como o silte, argila, sílica, coloides e matéria orgânica.

O modelo para estimar a transparência apresentou desempenho moderado para os parâmetros $R^{2}$ de $0,69, r$ de 0,83 , ficando abaixo do valor considerado satisfatório, de 0,75 para o índice de Willmott (d), com valor de 0,68 , e, foi considerado adequado para o NSE 0,44 (Figura 6C). O modelo apresenta erro de estimativa com EMA de 0,21 e REMQ de 0,31 m. A estimativa da transparência tende a superestimar os valores indicativos de baixa transparência e subestimar os de transparência acima de $1,20 \mathrm{~m}$.

O modelo para a variável condutividade elétrica apresentou $\mathrm{R}^{2}$ de $0,72, \mathrm{r}$ de 0,85 , ambos classificados como moderado, índice de Willmott (d) de 0,53 , abaixo do valor considerado satisfatório $(0,75)$ e NSE de 0,76 , considerado satisfatório (Figura 6D). O modelo apresenta baixo erro de estimativa com EMA de 0,0217 e RQEM de 0,0224 (dS m $\left.{ }^{-1}\right)$. Apesar de o modelo subestimar as concentrações de condutividade elétrica, apresenta a mesma tendência, ou seja, de subestimar os valores menores e maiores de condutividade elétrica da água.

Verifica-se pelos índices aplicados, que os modelos ajustados apresentam desempenhos aceitáveis. Os mesmos apresentam relação significativa estatisticamente a 0,01 , entre os dados medidos e estimados (Figura 6). Observa-se que os modelos desenvolvidos para as variáveis limnológicas (Figura 4), mostraram-se confiáveis, indicando que estas variáveis podem ser quantificadas remotamente a partir dos dados de sensoriamento remoto de campo. 


\section{Conclusões}

1. Os modelos desenvolvidos para as variáveis limnológicas, sólidos inorgânicos suspensos, turbidez, transparência e condutividade elétrica, mostraram-se confiáveis, indicando que estas variáveis podem ser quantificadas remotamente a partir dos dados de sensoriamento remoto de campo.

2. É possível a avaliação e o monitoramento da qualidade da água de sistemas aquáticos da região semiárida a partir de dados de sensoriamento remoto, através de dados espectrais in situ.

3. Em geral, os algoritmos empíricos usados para estimativa das concentrações de COAs e dados limnológicos por meio de dados de sensoriamento remoto hiperespectrais de campo são estabelecidos somente com auxílio de dados de verdade de campo e são úteis apenas dentro do contexto a partir do qual foram determinados.

4. Portanto, é preciso que os estudos avancem no sentido de estabelecer outras regressões empíricas e modelos semianalíticos, visando atender aos intervalos específicos de variação das concentrações dos COAs e atributos limnológicos.

\section{Agradecimentos}

Ao Instituto Nacional de Ciência e Tecnologia em Salinidade - INCTSal, a Coordenação de Aperfeiçoamento de Pessoal de Nível Superior - CAPES e ao Conselho Nacional de Desenvolvimento Científico e Tecnológico - CNPq pelo apoio financeiro à pesquisa.

\section{Literatura Citada}

Alexandre, D. M. B.; Andrade, E. M.; Lopes, F. B; Palácio, H. A. Q.; Ferreira, A.C. S. The water quality investigation using GIS and multivariable analysis in a semiarid region reservoir. Revista Ciência Agronômica, v.41, p.554-561, 2010.

Barros, R. S.; Cruz, C. B. M.; Medeiros, A. F. F.; Seabra, V. S. Estimativa de turbidez e temperatura da água a partir de dados dos sensores TM e ETM+ para a Baía de Guanabara. In: Simpósio Brasileiro de Sensoriamento Remoto, 2003, Belo Horizonte. Anais... Belo Horizonte: INPE, 2003. p.2435-2442.

Chen, Z.; Curran, P. J.; Hanson, J. D. Derivative refrectance spectroscopy to estimate suspended sediment concentration. Remote Sensing of Enviromment, v.40, p.67-77, 1992.

Choubey, V. K. Monitoring surface water conductivity with Indian remote sensing satellite data: A case study from central India. Hydrological, Chemical and Biological Processes of Transformation and Transport of Contaminants in Aquatic Environments (Proceedings of the Rostov-onDon Symposium). IAHS Publication, n.219, p.317-326. 1994.

Dekker, A. G.; Malthus, T. J.; Wijnen, M. M. Spectral band location for remote sensing of turbid and/or eutrophic waters. Thematic Conference on Remote Sensing, 1, 1992, Emerging Technologies and Systems. Proceedings... Ann Arbor: Environmental Research Institute of Michigan. 1992. p.955-970.
DNOCS - Departamento Nacional de Obras Contra as Secas. Barragem Orós. <http://www.dnocs.gov.br/ dnocs/doc/ canais/barragens/Barragem\%20do\%20Ceara/oros.htm>. 12 Jan. 2014.

Gitelson, A. A.; Gao, B.; Li, R.; Berdnikov, S.; Saprygin, V. Estimation of chlorophyll-a concentration in productive turbid waters using a Hyperspectral Imager for the Coastal Ocean - the Azov Sea case study. Environmental Research Letters. v.6, p.1-7, 2011.

Guedes, H. A. S.; Silva, D. D.; Elesbon, A. A. A.; Ribeiro, C. B. M.; Matos, A. T.; Soares, J. H. P. Aplicação da análise estatística multivariada no estudo da qualidade da água do Rio Pomba, MG. Revista Brasileira de Engenharia Agrícola e Ambiental, v.16, p.558-563, 2012.

Han, L.; Rundquist, D.C. Comparison of NIR/RED ratio and first derivative of reflectance in estimating algal-chlorophyll concentration: a case study in a Turbid Reservoir. Remote Sensing of Environment, v.62, p.255-261, 1997.

Jong, J.; Rooy, P. T. J. C.; Hosper, S. H. Living with water: at the cross-roads of change. Water Science Technology, v.8, p.393-400, 1995.

Le, C.; Hu, C.; Cannizzaro, J.; English, D.; Muller-Karger, F.; Lee, Z. Evaluation of chlorophyll-a remote sensing algorithms for an optically complex estuary. Remote Sensing of Environment. v.129, p.75-89, 2013.

Li, S.; Li, J.; Zhang, Q. Water quality assessment in the rivers along the water conveyance system of the Middle Route of the South to North Water Transfer Project (China) using multivariate statistical techniques and receptor modeling. Journal of Hazardous Materials, v.95, p.306317, 2011.

Lodhi, M. A.; Rundquist, D. C.; Han, L.; Kuzila, M. S. The Potential for Remote Sensing of Loess Soils Suspended in Surface Waters. Journal of the American Water Resources Association. v.33, p.111-117, 1997.

Lopes, F. B.; Sales, A. G. C.; Chaves, L. C. G.; Meireles, A. C. M.; Andrade, E. M. Modelos para estimativas de sedimentos em suspensão e da condutividade elétrica da água usando dados de sensoriamento remoto. In: Congresso Brasileiro de Engenharia Agrícola - CONBEA, 42, 2013. Anais... Fortaleza: SBEA, 2013. p.1-10.

Machado, R. E.; Vettorazzi, C. A.; Cruciani, D. E. Simulação de escoamento em uma microbacia hidrográfica utilizando técnicas de modelagem e geoprocessamento. Revista Brasileira de Recursos Hídricos, v.8, p.147-155, 2003.

Milton, E. J. Principles of field spectroscopy. International Journal of Remote Sensing, v.8, p.1807-1827, 1987.

Milton, J. S. Statistical methods in the biological and health sciences. 2.ed. New York: McGrow-Hill, 1992. 526p.

Nash, J. E.; Sutcliffe, J. V.: River flow forecasting through conceptual models, Part I - A discussion of principles, Journal of Hydrology, v.10, p.282-290, 1970.

Norusis, M. J. SPSS Base System User's Guide. Chicago: SPSS Inc., $1990.520 \mathrm{p}$. 
Novo, E. M. L. M. Sensoriamento remoto aplicado à ecologia aquática In: Roland, F.; César, D.; Marinho, M. (ed.). Lições de limnologia. São Carlos: RiMa, 2005. cap.5, p.417-432.

Pereira, A. C. F.; Galo, M. L. B. T.; Velini, E. D. Inferência da transparência da água - reservatório de Itupararanga/ SP, a partir de imagens multiespectrais IKONOS e espectrorradiometria de campo. Revista Brasileira de Cartografia, n.63/01, p.179-190, 2011.

Rudorff, C. M.; Novo, E. M. L. M.; Galvão, L. S.; Filho, W. P. Análise derivativa de dados hiperespectrais medidos em nível de campo e orbital para caracterizar a composição de águas opticamente complexas na Amazônia. Acta Amazonica, v.37, p.279-290, 2007.
Silva, A. P. S.; Dias, H. C. T.; Bastos, R. K. X.; Silva E. Qualidade da água do reservatório da Usina Hidrelétrica (UHE) de Peti, Minas Gerais. Revista Árvore, v.33, p.1063-1069, 2009.

UNESCO - Organização das Nações Unidas para a Educação, a Ciência e a Cultura. Relatório Mundial das Nações UNIDAS Sobre o Desenvolvimento dos Recursos Hídricos 4. <http:// unesdoc.unesco.org/images/0021/002154/215491por.pdf $>$. 10 Out. 2012.

Willmott, C. J.; Ackleson, S. G.; Davis, R. E.; Feddema, J. J.; Klink, K. M.; Legates, D. R.; Rowe, C. M.; O’donnell, J. Statistics for the evaluation and comparison of models. Journal of Geophysical Research, v.90, p.8995-9005, 1985. 